\title{
GEVREY CLASS REGULARITY OF THE MAGNETOHYDRODYNAMICS EQUATIONS
}

\author{
SANGJEONG KIM ${ }^{1}$
}

(Received 3 October, 1998; revised 30 August, 1999)

\begin{abstract}
In this article. we use the method of Foias and Temam to show that the strong solutions of the time-dependent magnetohydrodynamics equations in a periodic domain are analytic in time with values in a Gevrey class of functions. As immediate corollaries we find that the solutions are analytic in $H^{r}$-norms and that the solutions become smooth immediately after the initial time.
\end{abstract}

\section{Introduction}

The non-dimensional form of the magnetohydrodynamics (MHD) equations is

$$
\begin{aligned}
\frac{\partial}{\partial t} u+(u \cdot \nabla) u-\frac{1}{R_{e}} \Delta u+S \nabla\left(\frac{B^{2}}{2}\right)-S(B \cdot \nabla) B & =f \\
\frac{\partial}{\partial t} B+(u \cdot \nabla) B-(B \cdot \nabla) u+\frac{1}{R_{m}} \operatorname{curl}(\operatorname{curl} B) & =0 \\
\operatorname{div} u=\operatorname{div} B & =0
\end{aligned}
$$

where $u=\left(u_{1}(x, t), u_{2}(x, t), u_{3}(x, t)\right)$ is the velocity of the particle of fluid which is at point $x$ at time $t, B=\left(B_{1}(x, t), B_{2}(x, t), B_{3}(x, t)\right)$ is the magnetic field at point $x$ at time $t, f=f(x, t)$ is a volume density force, $R_{e}$ is the Reynolds number, $R_{m}$ is the magnetic Reynolds number and $S=M^{2} /\left(R_{e} R_{m}\right)$, where $M$ is the Hartan number.

These equations are important in the physics of plasma. The existence of weak and strong solutions and some regularities have been established by Sermange and Temam [3].

In this paper, we will consider the Gevrey class regularity of MHD equations in a periodic domain in $\mathbb{R}^{N}$ with $N=2$ or 3 following the method used by Foias and

\footnotetext{
'Korean Minjok Leadership Academy, 1334, Sosa, Anheung, Hoengsung, Kangwon, 225-823, Korea; e-mail: urchin@minjok.hs.kr.

(C) Australian Mathematical Society 2002, Serial-fee code $0334-2700 / 02$
} 
Temam [1]. In that paper Foias and Temam showed that the strong solutions of the Navier-Stokes equations in a periodic domain are analytic in time with the values in a Gevrey class of functions. We will prove a similar result for the MHD equations using a similar method.

The organization of this paper is as follows. In Section 2, we introduce some function spaces. In Section 3, we introduce known results about existence, uniqueness and regularity. In Section 4, we prove the main theorem, namely we establish Gevrey class regularity and derive some related regularity properties.

\section{Function spaces}

We supplement the system (1.1)-(1.3) with the following initial and boundary conditions:

$$
\begin{aligned}
u(x, 0) & =u_{0}(x), & B(x, 0) & =B_{0}(x), \\
u\left(x+L \mathrm{e}_{i}, t\right) & =u(x, t), & B\left(x+L \mathrm{e}_{i}, t\right) & =B(x, t),
\end{aligned}
$$

for all $x \in \mathbb{R}^{N}$ and $t>0$, where $L$ is the period and $\left\{\mathrm{e}_{i}\right\}_{i=1}^{N}$ is an orthonormal basis of the space. But we will regard $L$ to be $2 \pi$ for notational simplicity. When the dimension of the space is $N=2$, we classically define the operators curl $u=\partial u_{2} / \partial x_{1}-\partial u_{1} / \partial x_{2}$ for every vector function $u=\left(u_{1}, u_{2}\right)$ and $\widetilde{\operatorname{curl}} \phi=\left(\partial \phi / \partial x_{2},-\partial \phi / \partial x_{1}\right)$ for every scalar function $\phi$. We recall the two-dimensional formula

$$
\widetilde{\text { curl }} \operatorname{curl} u=\operatorname{grad} \operatorname{div} u-\Delta u,
$$

which corresponds to the three-dimensional formula

$$
\text { curl curl } u=\operatorname{grad} \operatorname{div} u-\Delta u \text {. }
$$

The two-dimensional MHD equations are (1.1)-(1.3) with the term $\widetilde{\operatorname{curl}}(\mathrm{curl} B)$ replacing curl(curl $B)$. Thus if $\operatorname{div} u=0$, then $\operatorname{curl} \operatorname{curl} u=-\Delta u$.

Let $T>0$ and let $X$ be a Banach space. We shall consider $L^{p}(0, T ; X), 1 \leq p \leq \infty$, which is the space of functions from $[0, T]$ into $X$, which are $L^{p}$ for the Lebesgue measure $d t$. This is a Banach space for the norm

$$
\left(\int_{0}^{T}\|u(t)\|_{X}^{p} d t\right)^{1 / p} \quad \text { for } 1 \leq p<\infty, \quad \underset{0 \leq I \leq T}{\operatorname{ess} \sup }\|u(t)\|_{X} \quad \text { for } p=\infty .
$$

We denote by $L^{2}(\mathscr{Q})$ the space of $\mathbb{R}^{N}$-valued functions on $\mathscr{Q}$ which are square integrable for the Lebesgue measure $d x=d x_{1} \cdots d x_{N}$. This is a Hilbert space for the scalar product

$$
(u, v)=\int_{\mathscr{Q}} u(x) \cdot v(x) d x
$$


Using Fourier series expressions we can identify $L^{2}(\mathscr{Q})$ with the space of functions $u$ satisfying

$$
u=\sum_{j \in \mathbb{Z}^{N}} u_{j}(t) e^{i j \cdot x}, \quad u_{j}(t) \in \mathbb{C}^{N}, u_{-j}=\bar{u}_{j} \quad \text { for } t \in[0, T] .
$$

For $m \in \mathbb{N}$, we also introduce

$$
H^{m}(\mathscr{Q})=\left\{\left.u \in L^{2}(\mathscr{Q})\left|(2 \pi)^{N} \sum_{j \in \mathbb{Z}^{N}}\right| j\right|^{2 m}\left|u_{j}\right|^{2}<\infty, u_{0}=0\right\}
$$

and $H^{-m}(\mathscr{Q})$, the dual space of $H^{m}(\mathscr{Q})$. Also

$$
\begin{aligned}
V & =\left\{u \in H^{1}(\mathscr{Q}) \mid j \cdot u_{j}=0 \text { for all } j \in \mathbb{Z}^{N}\right\}, \\
H & =\left\{u \in H^{0}(\mathscr{Q}) \mid j \cdot u_{j}=0 \text { for all } j \in \mathbb{Z}^{N}\right\}
\end{aligned}
$$

and $V^{\prime}$ is the dual space of $V$. We equip $V$ with the scalar product

$$
((u, v))=\sum_{k=1}^{N}\left(\frac{\partial u}{\partial x_{k}}, \frac{\partial v}{\partial x_{k}}\right)=\sum_{j \in \mathbb{Z}^{N}}(2 \pi)^{N}|j|^{2} u_{j} \cdot \bar{v}_{j}
$$

which is a scalar product on $H^{1}(\mathscr{Q})$.

We also introduce the spaces $\nabla$ and $B$ :

$$
\vee=\{(u, B) \mid u, B \in V\}, \quad \mathbb{H}=\{(u, B) \mid u, B \in H\} .
$$

We equip ㄴo with the scalar products

$$
(\Phi, \Psi)=(u, v)+(B, C) \text { for all } \Phi=(u, B), \Psi=(v, C) \in \mathbb{W}
$$

providing a norm on $\mathbb{N},|\Phi|=\{(\Phi, \Phi)\}^{1 / 2}$. We also equip $\mathbb{V}$ with the scalar products

$$
((\Phi, \Psi))=((u, v))+((B, C))
$$

providing a norm on $\mathbb{V},\|\Phi\|=\{((\Phi, \Phi))\}^{1 / 2}$.

We define an operator $\mathscr{A} \in \mathscr{L}\left(V, V^{\prime}\right)$ to be such that

$$
\langle\mathscr{A} u, v\rangle=((u, v)) \quad \text { for all } u, v \in V .
$$

We also consider $\mathscr{A}$ as an unbounded operator on $H$ whose domain is

$$
\mathscr{D}(\mathscr{A})=\{u \in V, \mathscr{A} u \in H\}=H^{2} \cap V .
$$


Since we consider divergence-free functions on a periodic domain, $\mathscr{A}$ is actually $-\Delta$. And since $\mathscr{A}$ is a nonnegative symmetric functional, we can define $\mathscr{A}^{\alpha}$ and the domain of $\mathscr{A}^{\alpha}$ is the set of functions $u$ such that

$$
(2 \pi)^{N} \sum_{j \in \mathbb{Z}^{N}}|j|^{4 \alpha}\left|u_{j}\right|^{2}=\left|\mathscr{A}^{\alpha} u\right|^{2}<\infty .
$$

For $\tau>0$ given, we consider also the Gevrey class $\mathscr{D}\left(e^{\tau \mathbb{A}^{1 / 2}}\right)$, that is, the set of functions $u$ satisfying

$$
(2 \pi)^{N} \sum_{j \in \mathbb{Z}^{N}} e^{2 \tau i j \mid}\left|u_{j}\right|^{2}=\left|e^{\tau \infty^{1 / 2}} u\right|^{2}<\infty
$$

with inner product

$$
(u, v)_{\tau}=(2 \pi)^{N} \sum_{j \in \mathbb{Z}^{N}} e^{2 \tau|v|} u_{j} \cdot \bar{v}_{j}
$$

with norm $|u|_{\tau}=\left\{(2 \pi)^{N} \sum_{j \in \mathbb{Z}^{N}} e^{2 \tau|j|}\left|u_{j}\right|^{2}\right\}^{1 / 2}$. And for $\mathscr{D}\left(\mathscr{A}^{1 / 2} e^{\tau \mathscr{A}^{1 / 2}}\right)$, we equip the inner product

$$
((u, v))_{\tau}=(2 \pi)^{N} \sum_{j \in \mathbb{Z}^{N}}|j|^{2} e^{2 \tau|j|} u_{j} \cdot \bar{v}_{j}
$$

with the norm $\|u\|_{\tau}=\left\{(2 \pi)^{N} \sum_{j \in \mathbb{Z}^{N}}|j|^{2} e^{2 \tau i v}\left|u_{j}\right|^{2}\right\}^{1 / 2}$.

LEMMA 2.1. Suppose $|u|_{\tau}^{2}=(2 \pi)^{N} \sum_{j \in \mathbb{Z}^{N}} e^{2 \tau i \dot{ } \mid}\left|u_{j}\right|^{2}<\infty$ for some $\tau>0$. Then $u \in \mathscr{C}^{\infty}$.

ProOF. Since $(2 \tau|j|)^{k} / k !<e^{2 \tau|j|}$ for all $j \in \mathbb{Z}^{N}$ and $k \in \mathbb{N}$,

$$
(2 \pi)^{N} \sum_{j \in \mathbb{Z}^{N}}|j|^{2 k}\left|u_{j}\right|^{2}=\|u\|_{H^{k}}^{2}<\infty .
$$

Thus $u \in \bigcap_{k=1}^{\infty} H^{k}$. By Sobolev imbedding, we get $u \in \mathscr{C}^{\infty}$.

We define now a trilinear form on $L^{1}(\mathscr{Q}) \times W^{1.1}(\mathscr{Q}) \times L^{1}(\mathscr{Q})$ by setting

$$
b(u, v, w)=\sum_{i, j=1}^{N} \int_{\mathscr{Q}} u_{i} D_{i} v_{j} w_{j} d x \quad\left(\text { where } D_{i}=\partial / \partial x_{i}\right),
$$

whenever the integrals make sense. We know the trilinear form $b$ is continuous on $\left(H^{1}(\mathscr{Q})\right)^{3}[4]$. Thus we can define a continuous bilinear operator $\mathscr{B}$ from $V \times V$ into $V^{\prime}$ with $\langle\mathscr{B}(u, v), w\rangle=b(u, v, w)$. 


\section{Known results}

Let $T>0$ be given and let us assume that $(p, u, B)$ is a smooth solution of (1.1)-(2.2). Multiplying (1.1) by a test function $v \in V$ and integrating over $\mathscr{Q}$, we obtain

$$
\frac{\partial}{\partial t}(u, v)+\frac{1}{R_{e}}((u, v))+b(u, u, v)-S b(B, B, v)=(f, v) .
$$

We also multiply (1.2) by a test function $C \in V$ and integrate over $\mathscr{Q}$, then

$$
\frac{\partial}{\partial t}(B, C)+\frac{1}{R_{m}}((B, C))+b(u, B, C)-b(B, u, C)=0 .
$$

Thus we define a strong solution of the MHD equations.

DEFINITION 3.1 (Strong solution). Assume $N=2$ or $3, f \in L^{2}(0, T ; H)$ and $\Phi_{0}=\left(u_{0}, B_{0}\right)$ is given in $\mathbb{V}$. Then $\Phi=(u, B)$ is a strong solution of the MHD equations if $u, B \in L^{2}(0, T ; \mathscr{D}(\mathscr{A})) \cap L^{\infty}(0, T ; V)$ and $\Phi$ satisfies (3.1), (3.2) for all $\Psi=(v, C) \in \mathbb{V}$.

Using operators $\mathscr{A}$ and $\mathscr{B}$, the previous equations, (3.1) and (3.2), may be written as

$$
\begin{aligned}
& \frac{\partial u}{\partial t}+\frac{1}{R_{e}} \mathscr{A} u+\mathscr{B}(u, u)-S \mathscr{B}(B, B)=f, \\
& \frac{\partial B}{\partial t}+\frac{1}{R_{m}} \mathscr{A} B+\mathscr{B}(u, B)-\mathscr{B}(B, u)=0 .
\end{aligned}
$$

The following result about existence and uniqueness is known [3].

THEOREM 3.2. Let $f ; u_{0}, B_{0}$ be given with $f \in L^{\infty}(0, T ; H), \Phi_{0}=\left(u_{0}, B_{0}\right) \in \mathbb{V}$. (1) If $N=2$, the strong solution $\Phi=(u, B)$ of the MHD equations uniquely exists and satisfies

$$
\Phi \in L^{2}(0, T ; \mathscr{D}(\mathscr{A})) \cap L^{\infty}(0, T ; \vee) .
$$

(2) If $N=3$, there exists $T_{*}>0$ (depending on $\left.\Omega, f,\|\Phi\|\right)$ such that there exists a unique strong solution $\Phi$ on $\left[0, T_{*}\right]$, which satisfies (3.5) with $T$ replaced by $T_{*}$.

\section{Gevrey class regularity}

LEMMA 4.1. Let $u, v, w$ be given in $\mathscr{D}\left(\mathscr{A} e^{\tau \mathbb{A}^{1 / 2}}\right), \tau>0$. Then the following inequalities hold in space dimension $N=2$ or 3 :

$$
\left|e^{\tau \sin ^{1 / 2} \mathscr{B}(u, v)}\right|_{L^{2}}^{2} \leq c\|u\|_{\tau}|\mathscr{A} u|_{\tau}\|v\|_{\tau}^{2},
$$




$$
\left|\left(e^{\tau \mathscr{A}^{1 / 2}} \mathscr{B}(u, v), \mathscr{A} e^{\tau \mathscr{A}^{1 / 2}} w\right)_{L^{2}}\right| \leq c\|u\|_{\tau}^{1 / 2}|\mathscr{A} u|_{\tau}^{1 / 2}\|v\|_{\tau}|\mathscr{A} w|_{\tau}
$$

where $c>0$ is independent of $u, v, w, \tau$.

ProOF. We set $u=\sum_{j \in \mathbb{Z}^{N}} u_{j} e^{i j \cdot x}, u^{*}=\sum_{j \in \mathbb{Z}^{N}} u_{j}^{*} e^{i j \cdot x}, u_{j}^{*}=e^{\tau|j|} u_{j}$ and use similar notation for $v$ and $w$. We have $(\mathscr{B}(u, v), w)=(2 \pi)^{N} i \sum_{j+k=l}\left(u_{j} \cdot k\right)\left(v_{k} \cdot \bar{w}_{l}\right)$. Also

$$
\begin{aligned}
\left(e^{\tau \mathscr{A}^{1 / 2}} \mathscr{B}(u, v), \mathscr{A} e^{\tau \mathscr{A}^{1 / 2}} w\right) & =(2 \pi)^{N} i \sum_{j+k=l}\left(u_{j} \cdot k\right)\left(v_{k} \cdot \bar{w}_{l}\right)|l|^{2} e^{2 \tau|l|} \\
& =(2 \pi)^{N} i \sum_{j+k=l}\left(u_{j}^{*} \cdot k\right)\left(v_{k}^{*} \cdot \bar{w}_{l}^{*}\right)|l|^{2} e^{\tau(|l|-|j|-|k|)} .
\end{aligned}
$$

Now since $|l|-|j|-|k|=|j+k|-|j|-|k| \leq 0$, we have

$$
\left|\left(e^{\tau \mathscr{A}^{1 / 2}} \mathscr{B}(u, v), \mathscr{A} e^{\tau \mathscr{A}^{1 / 2}} w\right)\right| \leq(2 \pi)^{N} \sum_{j+k=l}\left|u_{j}^{*}\right|\left|k \| v_{k}^{*}\right|\left|w_{l}^{*}\right||l|^{2} .
$$

Then the right-hand side of (4.1) is equal to the integral $\int_{\mathscr{Q}} \xi(x) \psi(x) \theta(x) d x$, where $\xi(x)=\sum_{j \in \mathbb{Z}^{N}}\left|u_{j}^{*}\right| e^{i j \cdot x}, \psi(x)=\sum_{k \in \mathbb{Z}^{N}}|k|\left|v_{k}^{*}\right| e^{i k \cdot x}, \theta(x)=\sum_{l \in \mathbb{Z}^{N}}|l|^{2}\left|w_{l}^{*}\right| e^{-i l \cdot x}$.

Assume $N=3$. Then by the Nirenberg inequality,

$|\xi|_{L^{\infty}} \leq c|D \xi|_{L^{2}}^{1 / 2}\left|D^{2} \xi\right|_{L^{2}}^{1 / 2}$

$$
\begin{aligned}
& \leq c\left(\sum_{j \in \mathbb{Z}^{N}}(2 \pi)^{N}|j|^{2}\left|u_{j}^{*}\right|^{2}\right)^{1 / 4}\left(\sum_{j \in \mathbb{Z}^{N}}(2 \pi)^{N}|j|^{4}\left|u_{j}^{*}\right|^{2}\right)^{1 / 4}=c\|u\|_{\tau}^{1 / 2}|\mathscr{A} u|_{\tau}^{1 / 2}, \\
|\psi|_{L^{2}}^{2} & =\sum_{k \in \mathbb{Z}^{N}}(2 \pi)^{N}|k|^{2}\left|v_{k}^{*}\right|^{2}=\|v\|_{\tau}^{2}, \quad|\theta|_{L^{2}}^{2}=\sum_{l \in \mathbb{Z}^{N}}(2 \pi)^{N}|l|^{4}\left|w_{l}^{*}\right|^{2}=|\mathscr{A} w|_{\tau}^{2} .
\end{aligned}
$$

Thus

$$
\begin{aligned}
\left|\left(e^{\tau \mathscr{A}^{1 / 2}} \mathscr{B}(u, v), \mathscr{A} e^{\tau \mathscr{A}^{1 / 2}} w\right)\right| & \leq\left|\int_{\mathscr{Q}} \xi(x) \psi(x) \theta(x) d x\right| \\
& \leq|\xi|_{L^{\infty}}|\psi|_{L^{2}}|\theta|_{L^{2}} \leq c\|u\|_{\tau}^{1 / 2}|\mathscr{A} u|_{\tau}^{1 / 2}\|v\|_{\tau}|\mathscr{A} w|_{\tau}
\end{aligned}
$$

Assume $N=2$. Then by the Nirenberg inequality,

$$
|\xi|_{L^{\infty}} \leq c|D \xi|_{L^{2}}=c\|u\|_{\tau} \leq c\|u\|_{\tau}^{1 / 2}|\mathscr{A} u|_{\tau}^{1 / 2}
$$

Thus

$$
\begin{aligned}
\left|\left(e^{\tau \mathscr{A}^{1 / 2}} \mathscr{B}(u, v), \mathscr{A} e^{\tau \mathscr{A}^{1 / 2}} w\right)\right| & \leq\left|\int_{\mathscr{Q}} \xi(x) \psi(x) \theta(x) d x\right| \\
& \leq|\xi|_{L^{\infty}}|\psi|_{L^{2}}|\theta|_{L^{2}} \leq c\|u\|_{\tau}^{1 / 2}|\mathscr{A} u|_{\tau}^{1 / 2}\|v\||\mathscr{A} w|_{\tau}
\end{aligned}
$$


And by similar calculation we obtain

$$
\begin{aligned}
& \left|\left(e^{\tau \mathscr{S}^{1 / 2}} \mathscr{B}(u, v), e^{\tau \mathscr{\infty}^{1 / 2}} \mathscr{B}(u, v)\right)\right| \\
& \quad \leq\left|\sum_{j+k=l+m}\left(u_{j} \cdot k\right)\left(v_{k} \cdot v_{m}\right)\left(u_{l} \cdot m\right) e^{\tau(|j+k|+|l+m|)}\right| \\
& \quad \leq \sum_{j+k=l+m}\left|u_{j} \| u_{l}\right||k||m|\left|v_{k}\right|\left|v_{m}\right| e^{\tau(j|+| k|+| l|+| m \mid)} \\
& \quad \leq c\|u\|_{\tau}|\mathscr{A} u|_{\tau}\|v\|_{\tau}^{2} .
\end{aligned}
$$

Now we obtain the following theorem.

THEOREM 4.2. Assume that $\left\|\Psi_{0}\right\| \leq M$ and $f \in L^{\infty}\left(0, T ; \mathscr{D}\left(e^{\sigma_{1} \infty^{1 / 2}}\right)\right)$ for some $\sigma_{1}>0$. Then there exists $T_{1}$ that depends on $f$ and $M$ such that the following holds:

(1) The strong solutions $\Psi$ of the MHD equations satisfy that $t \mapsto \mathscr{A}^{1 / 2} e^{\eta(t) \mathbb{S}^{1 / 2}} \Psi(t)$ is $L^{2}(\mathscr{Q})$-continuous for $t \in\left[0, T_{1}\right]$, where $\eta(t)=\min \left(t, \sigma_{1}\right)$.

(2) If the strong solutions $\Psi$ of the MHD equations satisfy $\|\Psi(t)\| \leq M$ on $\left[0, T_{0}\right]$, then $\Psi$ is analytic on $\left(\sigma, T_{0}+T_{1}\right)$ with respect to the norm of $\left(\mathscr{D}\left(\mathscr{A}^{1 / 2} e^{\sigma \mathcal{S}^{1 / 2}}\right)\right)^{2}$ for sufficiently small $\sigma>0$.

Proof. Let $\mathbb{C}$ denote the complex plane and $H_{\mathrm{C}}$ the complexified space of $H$, whose elements are denoted by $u+i v$, where $u, v \in H$; similarly $V_{\mathbf{C}}, V_{\mathbf{C}}^{\prime}$ are the complexified $V, V^{\prime}$. Let $\omega_{1}, \omega_{2}, \ldots$ be orthonormal eigenvectors in $V$ with respect to $\mathscr{A}$, whose eigenvalues are nondecreasing. Let $P_{m}$ be the projection onto $\left\langle\omega_{1}, \ldots, \omega_{m}\right\rangle$.

Consider now the complexified form of the Galerkin approximation of the MHD equations, that is, the complex differential system in $\left(P_{m} H_{\mathrm{C}}\right)^{2}$ :

$$
\begin{gathered}
\frac{d u_{m}}{d \zeta}(\zeta)+\frac{1}{R_{e}} \mathscr{A} u_{m}(\zeta)+P_{m} \mathscr{B}\left(u_{m}(\zeta), u_{m}(\zeta)\right)-S P_{m} \mathscr{B}\left(B_{m}(\zeta), B_{m}(\zeta)\right)=P_{m} f \\
\frac{d B_{m}}{d \zeta}(\zeta)+\frac{1}{R_{m}} \mathscr{A} B_{m}(\zeta)+P_{m} \mathscr{B}\left(B_{m}(\zeta), u_{m}(\zeta)\right)-P_{m} \mathscr{B}\left(u_{m}(\zeta), B_{m}(\zeta)\right)=0 \\
u_{m}(0)=P_{m}\left(u_{0}\right), \quad B_{m}(0)=P_{m}\left(B_{0}\right)
\end{gathered}
$$

where $\zeta \in \mathbb{C}$ and $u_{m}, B_{m}$ maps $\mathbb{C}$ (or an open subset of $\mathbb{C}$ ) into $P_{m} H_{\mathbb{C}}=\mathbb{C} w_{1}+$ $\cdots+\mathbb{C} w_{m}$. The complex differential system (4.2)-(4.4) possesses a unique analytic solution $u_{m}, B_{m}$ defined in some neighborhood of the origin. It is clear that the restriction $u_{m}, B_{m}$ to some interval $\left(0, T_{m}\right)$ of the real axis coincides with the Galerkin approximation $u_{m}(t), B_{m}(t)$ defined in the real field. 
We now get some estimates on $u_{m}, B_{m}$. Let $|\theta| \leq \pi / 4$, and $\eta(t)=\min \left(t, \sigma_{1}\right)$, $\zeta=s e^{i \theta}$. Then

$$
\begin{aligned}
\frac{1}{2} \frac{d}{d s} & \left\|u_{m}(\zeta)\right\|_{\eta(s \cos \theta)}^{2} \\
= & \operatorname{Re}\left[\frac{d}{d s} \eta(s \cos \theta)\left(\mathscr{A} u_{m}(\zeta), \mathscr{A}^{1 / 2} u_{m}(\zeta)\right)_{\eta(s \cos \theta)}+e^{i \theta}\left(\frac{d u_{m}}{d \zeta}, \mathscr{A} u_{m}\right)_{\eta(s \cos \theta)}\right] \\
\leq & \left|\mathscr{A} u_{m}(\zeta)\right|_{\eta(s \cos \theta)}\left\|u_{m}(\zeta)\right\|_{\eta(s \cos \theta)}+\operatorname{Re}\left[e ^ { i \theta } \left(-\left(1 / R_{e}\right) \mathscr{A} u_{m}(\zeta)\right.\right. \\
& \left.\left.-P_{m} \mathscr{B}\left(u_{m}(\zeta), u_{m}(\zeta)\right)+S P_{m} \mathscr{B}\left(B_{m}(\zeta), B_{m}(\zeta)\right)+P_{m} f, \mathscr{A} u_{m}(\zeta)\right)_{\eta(s \cos \theta)}\right] \\
\leq & \left|\mathscr{A} u_{m}(\zeta)\right|_{\eta(s \cos \theta)}\left\|u_{m}(\zeta)\right\|_{\eta(s \cos \theta)}-\frac{\cos \theta}{R_{e}}\left|\mathscr{A} u_{m}(\zeta)\right|_{\eta(s \cos \theta)}^{2} \\
& +c\left\|u_{m}(\zeta)\right\|_{\eta(s \cos \theta)}^{3 / 2}\left|\mathscr{A} u_{m}(\zeta)\right|_{\eta(s \cos \theta)}^{3 / 2} \\
& +S c\left\|B_{m}(\zeta)\right\|_{\eta(s \cos \theta)}^{3 / 2}\left|\mathscr{A} B_{m}(\zeta)\right|_{\eta(s \cos \theta)}^{1 / 2}\left|\mathscr{A} u_{m}(\zeta)\right|_{\eta(s \cos \theta)} \\
& +|f|_{\eta(s \cos \theta)}\left|\mathscr{A} u_{m}(\zeta)\right|_{\eta(s \cos \theta)} .
\end{aligned}
$$

We have

$$
\begin{aligned}
\frac{1}{2} \frac{d}{d s} \| & B_{m}(\zeta) \|_{\eta(s \cos \theta)}^{2} \\
= & \operatorname{Re}\left[\frac{d}{d s} \eta(s \cos \theta)\left(\mathscr{A} B_{m}(\zeta), \mathscr{A}^{1 / 2} B_{m}(\zeta)\right)_{\eta(s \cos \theta)}\right. \\
& \left.+e^{i \theta}\left(\frac{d}{d \zeta} B_{m}(\zeta), \mathscr{A} B_{m}(\zeta)\right)_{\eta(s \cos \theta)}\right] \\
\leq & \left|\mathscr{A} B_{m}(\zeta)\right|_{\eta(s \cos \theta)}\left\|B_{m}(\zeta)\right\|_{\eta(s \cos \theta)}+\operatorname{Re}\left[e ^ { i \theta } \left(-\left(1 / R_{m}\right) \mathscr{A} B_{m}(\zeta)\right.\right. \\
& \left.\left.-P_{m} \mathscr{B}\left(u_{m}(\zeta), B_{m}(\zeta)\right)+P_{m} \mathscr{B}\left(B_{m}(\zeta), u_{m}(\zeta)\right), \mathscr{A} B_{m}(\zeta)\right)_{\eta(s \cos \theta)}\right] \\
\leq & \left|\mathscr{A} B_{m}(\zeta)\right|_{\eta(s \cos \theta)}\left\|B_{m}(\zeta)\right\|_{\eta(s \cos \theta)}-\frac{\cos \theta}{R_{m}}\left|\mathscr{A} B_{m}(\zeta)\right|_{\eta(s \cos \theta)}^{2} \\
& +c\left\|u_{m}(\zeta)\right\|_{\eta(s \cos \theta)}^{1 / 2}\left|\mathscr{A} u_{m}(\zeta)\right|_{\eta(s \cos \theta)}^{1 / 2}\left\|B_{m}(\zeta)\right\|_{\eta(s \cos \theta)}\left|\mathscr{A} B_{m}(\zeta)\right|_{\eta(s \cos \theta)} \\
& +c\left\|B_{m}(\zeta)\right\|_{\eta(s \cos \theta)}^{1 / 2}\left|\mathscr{A} B_{m}(\zeta)\right|_{\eta(s \cos \theta)}^{3 / 2}\left\|u_{m}(\zeta)\right\|_{\eta(s \cos \theta)} .
\end{aligned}
$$

We introduce some norms for $\Phi=(u, B)$ as follows:

$$
[[\Phi]]_{\alpha}^{2}=\|u\|_{\alpha}^{2}+\|B\|_{\alpha}^{2}, \quad[\Phi]_{\alpha}^{2}=|u|_{\alpha}^{2}+|B|_{\alpha}^{2}
$$

Let $R=\min \left\{1 / R_{e}, 1 / R_{m}\right\}, \Phi_{m}=\left(u_{m}, B_{m}\right)$. Then we can rewrite the above inequalities as

$$
\frac{1}{2} \frac{d}{d s}\left[\left[\Phi_{m}\right]\right]_{\eta(s \cos \theta)}^{2}+R \cos \theta\left[\mathscr{A} \Phi_{m}\right]_{\eta(s \cos \theta)}^{2}
$$




$$
\begin{aligned}
\leq & c(3+S)\left[\left[\Phi_{m}\right]\right]_{\eta(s \cos \theta)}^{3 / 2}\left[\mathscr{A} \Phi_{m}\right]_{\eta(s \cos \theta)}^{3 / 2} \\
& +2\left[\left[\Phi_{m}\right]\right]_{\eta(s \cos \theta)}\left[\mathscr{A} \Phi_{m}\right]_{\eta(s \cos \theta)}+|f|_{\eta(s \cos \theta)}\left[\mathscr{A} \Phi_{m}\right]_{\eta(s \cos \theta)} \\
\leq & \frac{R \cos \theta}{2}\left[\mathscr{A} \Phi_{m}\right]_{\eta(s \cos \theta)}^{2}+\frac{c_{2}}{\cos ^{3} \theta}\left[\left[\Phi_{m}\right]\right]_{\eta(s \cos \theta)}^{6} \\
& +\frac{c_{3}}{\cos \theta}\left[\left[\Phi_{m}\right]\right]_{\eta(s \cos \theta)}^{2}+\frac{c_{4}}{\cos \theta}|f|_{\eta(s \cos \theta)}^{2}
\end{aligned}
$$

where $c_{2}, c_{3}, c_{4}$ depend on $R$ and $c$.

Since $|f|_{\eta(s \cos \theta)} \leq|f|_{\sigma_{1}}$, there exists $c_{5}$ which is dependent on $R, c$ and $|f|_{\sigma_{1}}$ such that

$$
\frac{d}{d s}\left[\left[\Phi_{m}\right]\right]_{\eta(s \cos \theta)}^{2}+R \cos \theta\left[\mathscr{A} \Phi_{m}\right]_{\eta(s \cos \theta)}^{2} \leq \frac{c_{5}}{\cos ^{3} \theta}\left(\left[\left[\Phi_{m}\right]\right]_{\eta(s \cos \theta)}^{2}+1\right)^{3} .
$$

Let $y(s)=\left[\left[\Phi_{m}\left(s e^{i \theta}\right)\right]\right]_{\eta(s \cos \theta)}^{2}+1$. Then for $s \leq T_{1}^{\prime}\left(\left\|\Phi_{0}\right\|\right)=3 \sqrt{2} /\left(32 c_{5}\left(1+\left\|\Phi_{0}\right\|^{2}\right)\right)$,

$$
1+\left[\left[\Phi_{m}\left(s e^{i \theta}\right)\right]\right]_{\eta(s \cos \theta)}^{2} \leq 2\left(1+\left\|\Phi_{0 m}\right\|^{2}\right) \leq 2\left(1+\left\|\Phi_{0}\right\|^{2}\right) .
$$

This shows that the solution of (4.2)-(4.4), which was defined and analytic in a neighbourhood of $\zeta=0$, actually extends to an analytic solution of this equation in an open set containing

$$
\mathscr{G}(M)=\left\{\zeta=s e^{i \theta}, 0<s<T_{1}^{\prime}(M),|\theta|<\pi / 4\right\} .
$$

The above estimates show that

$$
\sup _{\zeta \in \mathscr{G}(M)}\left\|\Phi_{m}(\zeta)\right\|_{\eta(s \cos \theta)}^{2} \leq 2\left(1+\left\|\Phi_{0}\right\|^{2}\right) .
$$

The analyticity of $\left(u_{m}, B_{m}\right)$ and Cauchy's integral formula allow us to deduce estimates on the derivatives of $\left(u_{m}, B_{m}\right)$ (with respect to $\zeta$ ) on a compact subset of $\mathscr{G}(M)$ from (4.8). For $0<\sigma<\sigma_{1}$, we denote $\mathscr{F}_{\sigma}(M)=\{z \in \mathbb{C}, z \in \mathscr{G}(M)$, $T_{*}>\operatorname{Re} z>\sigma$, where $T_{*}$ is derived from Theorem 3.2. Then for $0<\sigma<T_{1}^{\prime}(M)$, $\mathscr{F}_{\sigma}(M)$ is not empty. Let $\zeta \in \mathscr{F}_{\sigma}(M)$ and $k \in \mathbb{N}$. Then

$$
\begin{aligned}
\frac{d^{k} u_{m}}{d \zeta^{k}}(\zeta) & =\frac{k !}{2 \pi i} \int_{|z-\zeta|=d / 2} \frac{u_{m}(z)}{(z-\zeta)^{k+1}} d z, \\
\frac{d^{k} B_{m}}{d \zeta^{k}}(\zeta) & =\frac{k !}{2 \pi i} \int_{|z-\zeta|=d / 2} \frac{B_{m}(z)}{(z-\zeta)^{k+1}} d z,
\end{aligned}
$$

where $d=d\left(\zeta, \partial \mathscr{F}_{\sigma}(M)\right)$ is the distance of $\zeta$ to the boundary $\partial \mathscr{F}_{\sigma}(M)$. Therefore

$$
\left\|\frac{d^{k}}{d \zeta^{k}} u_{m}\right\|_{\sigma} \leq \frac{2^{k} k !}{d^{k}} \sup _{z \in \mathscr{F}_{\sigma}(M)}\left\|u_{m}(z)\right\|_{\sigma}, \quad\left\|\frac{d^{k}}{d \zeta^{k}} B_{m}\right\|_{\sigma} \leq \frac{2^{k} k !}{d^{k}} \sup _{z \in \mathscr{F}_{\sigma}(M)}\left\|B_{m}(z)\right\|_{\sigma} .
$$


Thus for any compact $K \subset \mathscr{F}_{\sigma}(M)$,

$$
\begin{aligned}
& \sup _{\zeta \in K}\left\|\frac{d^{k}}{d \zeta^{k}} u_{m}(\zeta)\right\|_{\sigma} \leq \frac{2^{k+1} k !}{\left[d\left(K, \partial \mathscr{F}_{\sigma}(M)\right)\right]^{k}}\left(1+\left\|\Phi_{0}\right\|^{2}\right)^{1 / 2}, \\
& \sup _{\zeta \in K}\left\|\frac{d^{k}}{d \zeta^{k}} B_{m}(\zeta)\right\|_{\sigma} \leq \frac{2^{k+1} k !}{\left[d\left(K, \partial \mathscr{F}_{\sigma}(M)\right)\right]^{k}}\left(1+\left\|\Phi_{0}\right\|^{2}\right)^{1 / 2} .
\end{aligned}
$$

On the other hand,

$$
\begin{aligned}
\frac{1}{R_{e}}\left|\mathscr{A} u_{m}\right|_{\sigma}^{2}= & \left(f, \mathscr{A} u_{m}\right)_{\sigma}+S\left(P_{m} \mathscr{B}\left(B_{m}, B_{m}\right), \mathscr{A} u_{m}\right)_{\sigma} \\
& -\left(P_{m} \mathscr{B}\left(u_{m}, u_{m}\right), \mathscr{A} u_{m}\right)_{\sigma}-\left(d u_{m} / d \zeta, \mathscr{A} u_{m}\right)_{\sigma} \\
\leq & |f|_{\sigma}\left|\mathscr{A} u_{m}\right|_{\sigma}+S c\left\|B_{m}\right\|_{\sigma}^{3 / 2}\left|\mathscr{A} B_{m}\right|_{\sigma}^{1 / 2}\left|\mathscr{A} u_{m}\right|_{\sigma} \\
& +c\left\|u_{m}\right\|_{\sigma}^{3 / 2}\left|\mathscr{A} u_{m}\right|_{\sigma}^{3 / 2}+\left|d u_{m} / d \zeta\right|_{\sigma}\left|\mathscr{A} u_{m}\right|_{\sigma}, \\
\frac{1}{R_{m}}\left|\mathscr{A} B_{m}\right|_{\sigma}^{2}= & \left(P_{m} \mathscr{B}\left(B_{m}, u_{m}\right), \mathscr{A} B_{m}\right)_{\sigma}-\left(P_{m} \mathscr{B}\left(u_{m}, B_{m}\right), \mathscr{A} B_{m}\right)_{\sigma} \\
& -\left(d B_{m} / d \zeta, \mathscr{A} B_{m}\right)_{\sigma} \\
\leq & c\left\|B_{m}\right\|_{\sigma}^{1 / 2}\left|\mathscr{A} B_{m}\right|_{\sigma}^{1 / 2}\left\|u_{m}\right\|_{\sigma}\left|\mathscr{A} B_{m}\right|_{\sigma} \\
& +\left.\left.c\left\|u_{m}\right\|_{\sigma}^{1 / 2}\left|\mathscr{A} u_{m}\right|_{\sigma}^{1 / 2}\left\|B_{m}\right\|_{\sigma}\right|_{\mathscr{A}} B_{m}\right|_{\sigma}+\left|d B_{m} / d \zeta\right|_{\sigma}\left|\mathscr{A} B_{m}\right|_{\sigma} .
\end{aligned}
$$

Thus

$$
\begin{aligned}
\frac{1}{R_{e}}\left|\mathscr{A} u_{m}\right|_{\sigma} & \leq|f|_{\sigma}+c(S+1)\left[\left[\Phi_{m}\right]\right]_{\sigma}^{3 / 2}\left[\mathscr{A} \Phi_{m}\right]_{\sigma}^{1 / 2}+\left[\left[d \Phi_{m} / d \zeta\right]\right]_{\sigma} \\
& \leq|f|_{\sigma}+R_{e} c^{2}(S+1)^{2}\left[\left[\Phi \Phi_{m}\right]\right]_{\sigma}^{3}+\frac{1}{4 R_{e}}\left[\mathscr{A} \Phi_{m}\right]_{\sigma}+\left[\left[d \Phi_{m} / d \zeta\right]\right]_{\sigma}, \\
\frac{1}{R_{m}}\left|\mathscr{A} B_{m}\right|_{\sigma} & \leq 2 c\left[\left[\Phi_{m}\right]\right]_{\sigma}^{3 / 2}[\mathscr{A} \Phi]_{\sigma}^{1 / 2}+\left\|d B_{m} / d \zeta\right\|_{\sigma} \\
& \leq 4 c^{2} R_{m}[[\Phi]]_{\sigma}^{3}+\frac{1}{4 R_{m}}[\mathscr{A} \Phi]_{\sigma}+\left\|d B_{m} / d \zeta\right\|_{\sigma} .
\end{aligned}
$$

Thus by (4.13) and (4.14),

$$
\begin{aligned}
{[\mathscr{A} \Phi]_{\sigma} \leq } & \left|\mathscr{A} u_{m}\right|_{\sigma}+\left|\mathscr{A} B_{m}\right|_{\sigma} \\
\leq & R_{e}|f|_{\sigma}+R_{e}^{2} c^{2}(S+1)^{2}\left[\left[\Phi_{m}\right]\right]_{\sigma}^{3}+\frac{1}{4}\left[\Phi_{m}\right]_{\sigma}+R_{e}\left[\left[d \Phi_{m} / d \zeta\right]\right]_{\sigma} \\
& +4 c^{2} R_{m}^{2}\left[\left[\Phi_{m}\right]\right]_{\sigma}^{3}+\frac{1}{4}\left[\mathscr{A} \Phi_{m}\right]_{\sigma}+R_{m}\left[\left[d \Phi_{m} / d \zeta\right]\right]_{\sigma} .
\end{aligned}
$$

Therefore

$$
\sup _{\zeta \in K}\left[\mathscr{A} \Phi_{m}(\zeta)\right]_{\sigma} \leq c_{6}(K)<\infty
$$


Again using Cauchy's formula and (4.15) we obtain also for every $\zeta \in K$ and $k \in N$

$$
\begin{aligned}
& \left|\mathscr{A} \frac{d^{k}}{d \zeta^{k}} u_{m}(\zeta)\right|_{\sigma} \leq \frac{2^{k} k !}{\left[d\left(K, \partial \mathscr{F}_{\sigma}(M)\right)\right]^{k}} \sup _{z \in K^{\prime}}\left|\mathscr{A} u_{m}(z)\right|_{\sigma}, \\
& \left|\mathscr{A} \frac{d^{k}}{d \zeta^{k}} B_{m}(\zeta)\right|_{\sigma} \leq \frac{2^{k} k !}{\left[d\left(K, \partial \mathscr{F}_{\sigma}(M)\right)\right]^{k}} \sup _{z \in K^{\prime}}\left|\mathscr{A} B_{m}(z)\right|_{\sigma} .
\end{aligned}
$$

Thus

$$
\sup _{\zeta \in K}\left[\mathscr{A} \frac{d^{k}}{d \zeta^{k}} \Phi_{m}(\zeta)\right]_{\sigma} \leq \frac{2^{k+1}}{\left[d\left(K, \partial \mathscr{F}_{\sigma}(M)\right)\right]^{k}} c_{6}\left(K^{\prime}\right),
$$

where $K^{\prime}=\left\{z \in \mathscr{F}_{\sigma}(M), d\left(z, \partial \mathscr{F}_{\sigma}(M)\right) \geq(1 / 2) d\left(K, \partial \mathscr{F}_{\sigma}(M)\right\}\right.$.

We now pass to the limit $m \rightarrow \infty$. For every compact subset of $\mathscr{F}_{\sigma}(M), \Phi_{m}$ is uniformly bounded in $\mathscr{D}\left(\mathscr{A} e^{\sigma \mathscr{A}^{1 / 2}}\right)$ and $d \Phi_{m} / d \zeta$ is also bounded in $\mathscr{D}\left(\mathscr{A} e^{\sigma \mathscr{A}^{1 / 2}}\right)$. Since $\left(\mathscr{A}^{1 / 2}\right)^{-1}$ is a compact operator, we can use the vector version of Montel's theorem, that is, there is a subsequence $\left\{\Phi_{m_{j}}\right\}$ which converges to a analytic function $\Phi$ in $\mathscr{D}\left(\mathscr{A}^{1 / 2} e^{\sigma \mathscr{A}^{1 / 2}}\right)$ on $\mathscr{F}_{\sigma}(M)$.

Since the restriction of $u_{m}, B_{m}$ to the real axis coincides with the Galerkin approximation in $R_{+}$of the MHD equations, it is clear that the restriction of $\Phi$ to the same interval $\left(0, T_{1}=\min \left\{T_{1}^{\prime}, T_{*}\right\}\right)$ of the real axis coincides with the unique solution of the MHD equations given by Theorem 3.2. Thus we obtain the real analyticity of $\Phi$ on the interval $\left(\sigma, T_{1}\right)$. Since $\|\Phi\| \leq M$ on $\left[0, T_{0}\right], \Phi$ is analytic in $\mathscr{D}\left(\mathscr{A}^{1 / 2} e^{\sigma \mathscr{A}^{1 / 2}}\right)$ for $t \in\left(\sigma, T_{0}+T_{1}\right)$.

Now we prove part (1) of Theorem 4.2.

By (4.5), $\mathscr{A} e^{\eta(s) \mathscr{A}^{1 / 2}} \Phi \in L^{2}\left(0, T_{1} ; \mathbb{H}\right)$. Thus

$$
\mathscr{A}^{1 / 2} e^{\eta(s) \mathscr{A}^{1 / 2}} \Phi \in L^{2}\left(0, T_{1} ; V\right) \text {. }
$$

By (4.16), $\mathscr{A}^{3 / 2} e^{\eta(s) \mathscr{A}^{1 / 2}} \Phi \in L^{2}\left(0, T_{1} ; \nabla^{\prime}\right)$. And if $v_{1}, v_{2}$ are $u$ or $B$, then by Lemma 4.1 and (4.5), we obtain that

$$
e^{\eta(s) \mathscr{A}^{1 / 2}} \mathscr{B}\left(v_{1}, v_{2}\right) \in L^{2}\left(0, T_{1} ; H\right) \quad \text { and } \quad \mathscr{A}^{1 / 2} e^{s \mathscr{S}^{1 / 2}} \mathscr{B}\left(v_{1}, v_{2}\right) \in L^{2}\left(0, T_{1} ; V^{\prime}\right)
$$

And since $f \in L^{\infty}\left(0, T ; \mathscr{D}\left(e^{\sigma_{1} \mathscr{A}^{1 / 2}}\right)\right)$, we obtain $\mathscr{A}^{1 / 2} e^{s \mathscr{A}^{1 / 2}} f \in L^{2}\left(0, T_{1} ; V^{\prime}\right)$.

Thus we obtain

$$
\frac{d}{d s}\left(\mathscr{A}^{1 / 2} e^{\eta(s) \mathscr{A}^{1 / 2}} u\right), \quad \frac{d}{d s}\left(\mathscr{A}^{1 / 2} e^{\eta(s) \mathscr{A}^{1 / 2}} B\right) \in L^{2}\left(0, T_{1} ; V^{\prime}\right)
$$

By (4.16), (4.17) we obtain $\mathscr{A}^{1 / 2} e^{\eta(s) \mathscr{A}^{1 / 2}} u, \mathscr{A}^{1 / 2} e^{\eta(s) \mathscr{A}^{1 / 2}} B \in \mathscr{C}\left(\left[0, T_{1}\right] ; L^{2}\right)$ [5, Ch. III, $\S 1.4]$.

COROLLARY 4.3. Under the same conditions as those of Theorem $4.2, \Psi$, the strong solution of the MHD equations, is analytic on $\left(0, T_{0}+T_{1}\right)$ with respect to the $H^{r}$-norm for $r \geq 0$. 
PRoOF. Let $t_{0} \in\left(0, T_{0}+T_{1}\right)$. Then there is $\sigma$ such that $0<\sigma<t_{0}$. Thus

$$
\frac{(2 \sigma)^{2 k}\left|\Psi(t)-\Psi\left(t_{0}\right)-\left(t-t_{0}\right) \frac{d}{d t} \Psi\left(t_{0}\right)\right|_{H^{k+1}}^{2}}{(2 k) !\left|t-t_{0}\right|^{2}} \leq \frac{\left[\left[\Psi(t)-\Psi\left(t_{0}\right)-\left(t-t_{0}\right) \frac{d}{d t} \Psi\left(t_{0}\right)\right]\right]_{\sigma}^{2}}{\left|t-t_{0}\right|^{2}} .
$$

By the above theorem, the right-hand side of the above inequality goes to zero. Thus the left-hand side of the above inequality also goes to zero. Thus $\Psi$ is analytic in $H^{k+1}$-norms. And $\Psi$ is analytic in $L^{2}$-norms by similar reasoning.

COROLlaRY 4.4. Under the same assumptions as those of Theorem 4.2, $\Phi(t) \in$ $\mathscr{C}^{\infty}(\mathscr{Q})$ with eventual modification on a set measure zero on $\left(0, T_{0}+T_{1}\right)$.

PROOF. If $t \in\left(0, T_{0}+T_{1}\right)$, then [ $\left.\Phi(t)\right]_{\sigma}$ is bounded for some $\sigma$ by the above theorem. Thus $\Phi(t)$ is smooth in $\mathbb{B}^{N}$ by Lemma 2.1 .

COROLLARY 4.5. If $N=2$, the strong solution is analytic on $(\sigma, T)$ with respect to the norm of $\left(\mathscr{D}\left(\mathscr{A}^{1 / 2} e^{\sigma \mathscr{A}^{1 / 2}}\right)\right)^{2}$ for sufficiently small $\sigma>0$.

PROOF. $\|\Phi\|$ is bounded on $(0, T)$ by Theorem 3.2. Thus we are done by the above theorem.

\section{Acknowledgments}

The author wishes to thank Professor Dongho Chae for suggesting the problem and for many helpful discussions.

\section{References}

[1] C. Foias and R. Temam, "Gevrey class regularity for the solutions of the Navier-Stokes equations", J. Funct. Anal. 68 (1988) 359-369.

[2] J. Lions and E. Magenes, Non-Homogeneous Boundary Value Problems and Applications (Springer, 1972).

[3] M. Sermange and R. Temam, "Some mathematical questions related to the MHD equations", Comm. Pure Appl. Math. XXXVI (1983) 635-664.

[4] R. Temam, Navier-Stokes Equations and Nonlinear Functional Analysis, CBMS-NSF Regional Conference Series in Applied Mathematics 41 (SIAM, Philadelphia, PA, 1983).

[5] R. Temam, Navier-Stokes Equations: Theory and Numerical Analysis (Elsevier Science Publishers B.V., 1985).

[6] R. Temam. Infinite-Dimensional Dynamical Systems in Mechanics and Physics, Volume 68 (Springer, 1988). 